\title{
Asymptotic Behavior of Solutions to the Finite-Difference Wave Equation
}

\author{
By Carl E. Pearson
}

\begin{abstract}
A stable finite-difference scheme for the wave equation may possess features not shared by the underlying partial differential equation. These discrepancies are explored; in particular, an asymptotic estimate for the magnitude of precursor effects is obtained.
\end{abstract}

1. Introduction. A natural finite-difference approximation for the wave equation

$$
u_{t t}=c^{2} u_{x x}
$$

$(c=$ constant $)$ is given by

$$
u_{j}^{n+1}-2 u_{j}^{n}+u_{j}^{n-1}=\alpha^{2}\left(u_{j+1}^{n}-2 u_{j}^{n}+u_{j-1}^{n}\right)
$$

where $u_{j}^{n}$ in Eq. (2) corresponds to $u(j \delta x, n \delta t)$ in Eq. (1), and where $\alpha=c \delta t / \delta x$. Here $\delta t$ and $\delta x$ are the time and space intervals, respectively. We consider the case $-\infty<x<\infty, t>0$. It was shown by Courant, Friedrichs, and Lewy in a wellknown paper [1] that if $u_{j}{ }^{0}$ and $u_{j}{ }^{1}$ are prescribed for all $j$, then the computational process represented by Eq. (2) will yield values for $u_{j}{ }^{n}$ which converge to the exact solution of Eq. (1) as $\delta x \rightarrow 0$ (for corresponding initial conditions), provided that the stability condition $\alpha<1$ is maintained. They point out that if $\alpha>1$, then the procedure (2) cannot possibly be convergent, because the domain of dependence of a point $(x, t)$ via Eq. (2) does not include all points of its domain of dependence via Eq. (1), and consequently a change in initial values near the endpoints of the domain of dependence will affect the solution of Eq. (1) but not that of Eq. (2). Since the proof of reference (1) is nonconstructive, it does not shed light on two aspects of the stable case $\alpha<1$. The first of these is that the speed with which signals propagate under Eq. (2) is $c / \alpha$, which in fact $\rightarrow \infty$ as $\alpha \rightarrow 0$; on the other hand, the signal speed associated with Eq. (1) is $c$. A second and related apparent paradox arises from the converse argument to that used by the above authors; since for $\alpha<1$ the domain of dependence for Eq. (2) includes points not in the domain of dependence for Eq. (1), it should be possible to alter the initial conditions so as to affect the solution of Eq. (2) but not that of Eq. (1). Of course, the fact that convergence does ensue as $\delta x \rightarrow 0$, for any choice of $\alpha<1$, demonstrates indirectly that these two apparent discrepancies must become unimportant in some sense as $\delta x \rightarrow 0$; nevertheless, it is of interest to demonstrate this result directly, and also to obtain explicit formulas for the errors.

We first obtain an exact solution of Eq. (2), corresponding to the initial conditions $u_{0}{ }^{0}=A, u_{0}{ }^{1}=A+B \delta t$ with $u_{j}{ }^{0}=u_{j}{ }^{1}=0$ for all $j \neq 0$. Any more general initial conditions can be constructed by means of a superposition of conditions of this form. We then consider the case $\alpha \ll 1$, which accentuates the apparent paradox, and use the saddle point method to obtain explicit asymptotic expressions for $u_{j}{ }^{n}$

Received November 8, 1968. 
valid for large values of $j$. In particular, a formula for the precursor signal is obtained, and it is confirmed that the speed with which a significant portion of energy is transmitted is indeed $c$. Our results are somewhat similar to those obtained for first-order equations by Kreiss and Lundquist [4] and by Apelkrans [5] who show that for dissipative difference schemes an error in initial data has an effect away from a characteristic which is of exponentially decaying order.*

2. Solution of Eq. (2). To solve Eq. (2) subject to the initial conditions

$$
\begin{aligned}
& u_{0}{ }^{0}=A, \quad u_{0}{ }^{1}=A+B \delta t \\
& u_{j}{ }^{0}=u_{j}{ }^{1}=0 \text { for } j \neq 0
\end{aligned}
$$

we define the generating function

$$
\varphi^{n}(z)=\sum_{-n+1}^{n-1} u_{j}^{n} z^{j}=\sum_{-\infty}^{\infty} u_{j}^{n} z^{j}
$$

where $z$ is a complex variable. The initial conditions (3) correspond to

$$
\varphi^{0}(z)=A, \quad \varphi^{1}(z)=A+B \delta t .
$$

Multiplying Eq. (2) by $z^{j}$ and summing over all $j$, we obtain a difference equation for $\varphi^{n}(z)$,

$$
\varphi^{n+1}-2\left[1+\left(\alpha^{2} / 2\right)(z+1 / z-2)\right] \varphi^{n}+\varphi^{n-1}=0
$$

whose solution, subject to the initial conditions (5), is

$$
\varphi^{n}(z)=\frac{(A+B \delta t)-A \rho_{-}}{\rho_{+}-\rho_{-}} \rho_{+}{ }^{n}+\frac{A \rho_{+}-(A+B \delta t)}{\rho_{+}-\rho_{-}} \rho_{-}{ }^{n}
$$

where

$$
\begin{aligned}
\rho_{ \pm} & =1+\frac{\beta^{2}}{n^{2}} \pm\left(2 \frac{\beta^{2}}{n^{2}}+\frac{\beta^{4}}{n^{4}}\right)^{1 / 2} \\
\beta & =\frac{c t}{\delta x}\left(\frac{1}{2}\left(z+\frac{1}{z}-2\right)\right)^{1 / 2} \\
t & =n \delta t .
\end{aligned}
$$

Holding $\delta x$ and $t$ (and therefore $\beta$ ) fixed, we now consider the case of large $n$; i.e., we permit $\alpha$ to become very small. An expansion in terms of $(1 / n)$ yields

$$
\begin{aligned}
\varphi^{n}(z)= & \frac{A}{2 \sqrt{ } 2}\left[e^{21 / 2 \beta}\left(2^{1 / 2}-\frac{\beta}{n}-\frac{1}{6} \frac{\beta^{3}}{n^{2}}+\cdots\right)\right. \\
& \left.+e^{-2^{1 / 2} \beta}\left(2^{1 / 2}+\frac{\beta}{n}+\frac{1}{6} \frac{\beta^{3}}{n^{2}}+\cdots\right)\right] \\
& +\frac{B t}{2(2)^{1 / 2} \beta}\left[e^{21 / 2 \beta}\left(1-\frac{1}{4} \frac{\beta^{2}}{n^{2}}-\frac{2^{1 / 2}}{12} \frac{\beta^{3}}{n^{2}}+\cdots\right)\right. \\
& \left.-e^{-2^{1 / 2} \beta}\left(1-\frac{1}{4} \frac{\beta^{3}}{n^{2}}+\frac{2^{1 / 2}}{12} \frac{\beta^{3}}{n^{2}}+\cdots\right)\right] .
\end{aligned}
$$

* It may be remarked that for $\alpha \ll 1$, our initial conditions correspond to a discontinuity in the data; this affects conventional error estimates. 
The value of $u_{j}^{n}$ can now be obtained by contour integration

$$
u_{j}^{n}=\frac{1}{2 \pi i} \int_{C} \frac{\varphi^{n}(z)}{z^{j+1}} d z
$$

where $C$ is any closed contour enclosing the origin. Up to terms of order $(1 / n)$, this gives

$$
\begin{aligned}
u_{j}^{n}=\frac{1}{2 \pi i} \int_{C} \frac{d z}{2 z^{j+1}}\left[A\left(e^{21 / 2 \beta}+e^{-21 / 2 \beta}\right)+\frac{B t}{2^{1 / 2} \beta}\left(e^{21 / 2 \beta}-e^{-21 / 2 \beta}\right)\right. & \\
& \left.-\frac{A \beta}{2^{1 / 2} n}\left(e^{21 / 2 \beta}-e^{-2^{1 / 2} \beta}\right)\right] .
\end{aligned}
$$

(We remark in passing that the first two terms correspond to the solution of

$$
\partial^{2} u_{j} / \partial t^{2}=(c / \delta x)^{2}\left[u_{j+1}-2 u_{j}+u_{j-1}\right]
$$

for a function $u_{j}(t)$, satisfying the initial conditions

$$
u_{0}(0)=A, \quad u_{0}^{\prime}(0)=B, \quad u_{j}(0)=u_{j}^{\prime}(0)=0 \text { for } j \neq 0 .
$$

With $z=e^{i \theta}$, Eq. (9) becomes

$$
\begin{aligned}
u_{j}^{n}=\frac{1}{2 \pi} \int_{0}^{2 \pi}\left[A \cos \left(\frac{2 c t}{\delta x} \sin \frac{\theta}{2}\right)+\frac{B \delta x}{2 c} \cdot \frac{\sin \left(\frac{2 c t}{\delta x} \sin \frac{\theta}{2}\right)}{\sin \frac{\theta}{2}}\right. \\
\left.+\frac{A c t}{n \delta x} \sin \frac{\theta}{2} \sin \left(\frac{2 c t}{\delta x} \sin \frac{\theta}{2}\right)\right] \cos j \theta d \theta \\
=A J_{2 j}\left(\frac{2 c t}{\delta x}\right)+\frac{B \delta x}{2 c} \int_{0}^{2 c t / \delta x} J_{2 j}(w) d w+\frac{A}{2 n} J_{2 j}^{\prime}\left(\frac{2 c t}{\delta x}\right)
\end{aligned}
$$

where various integral identities for $J_{p}$, the Bessel function of the first kind of order $p$, have been used.

Write $x=j \delta x$, and denote $c t / x$ by $\xi$. Equation (10) becomes

$$
u_{j}^{n}=A J_{2 j}(2 j \xi)+\frac{B \delta x}{2 c} \int_{0}^{2 j \xi} J_{2 j}(w) d w+\frac{A}{2 n} J_{2 j}^{\prime}(2 j \xi) .
$$

3. Asymptotic Behavior. Our purpose is to evaluate Eq. (11) asymptotically as $j$ becomes large, holding $\xi$ fixed. Note that, for the corresponding solution of Eq. (1), the conditions $\xi<1$ and $\xi>1$ represent the nonsignal and the signal zone, respectively. Except for the integral term in Eq. (11), the desired asymptotic expressions are readily available in the literature [2]. In Appendix I, the saddle point method is used to obtain an asymptotic expression for the integral term. Putting these results together, Eq. (11) becomes:

(a) for $\xi<1, \xi=\operatorname{sech} \lambda$

$$
u_{j}^{n} \sim \frac{e^{-2 j(\lambda-\tanh \lambda)}}{(4 \pi j \tanh \lambda)^{1 / 2}}\left[A\left(1+\frac{\sinh \lambda}{2 n}\right)+\frac{B \delta x}{2 c \sinh \lambda}\right],
$$


(b) for $\xi>1, \xi=\sec \lambda$

(13)

$$
\begin{aligned}
u_{j}^{n} \sim & \frac{A}{(\pi j \tan \lambda)^{1 / 2}}[\cos (2 j\{\tan \lambda-\lambda\}-\pi / 4) \\
\left.-\frac{\sin \lambda}{2 n} \sin (2 j\{\tan \lambda-\lambda\}-\pi / 4)\right] & \\
& +\frac{B \delta x}{2 c}\left[1-\frac{\cos (2 j\{\tan \lambda-\lambda\}+\pi / 4)}{(\pi j \tan \lambda)^{1 / 2} \sin \lambda}\right] .
\end{aligned}
$$

Equation (13) is easily seen to be compatible with the solution of Eq. (1) for the choice of initial conditions

$$
\begin{aligned}
& u(x, 0)=A, \quad u_{t}(x, 0)=B, \text { for } x \text { in }(-\delta x / 2, \delta x / 2), \\
& u(x, 0)=0, \quad u_{t}(x, 0)=0, \text { for all other } x .
\end{aligned}
$$

However, the point of present interest is that the precursor signal has the form given asymptotically by Eq. (12); since $\lambda>\tanh \lambda$, it is indeed seen to be exponentially small.

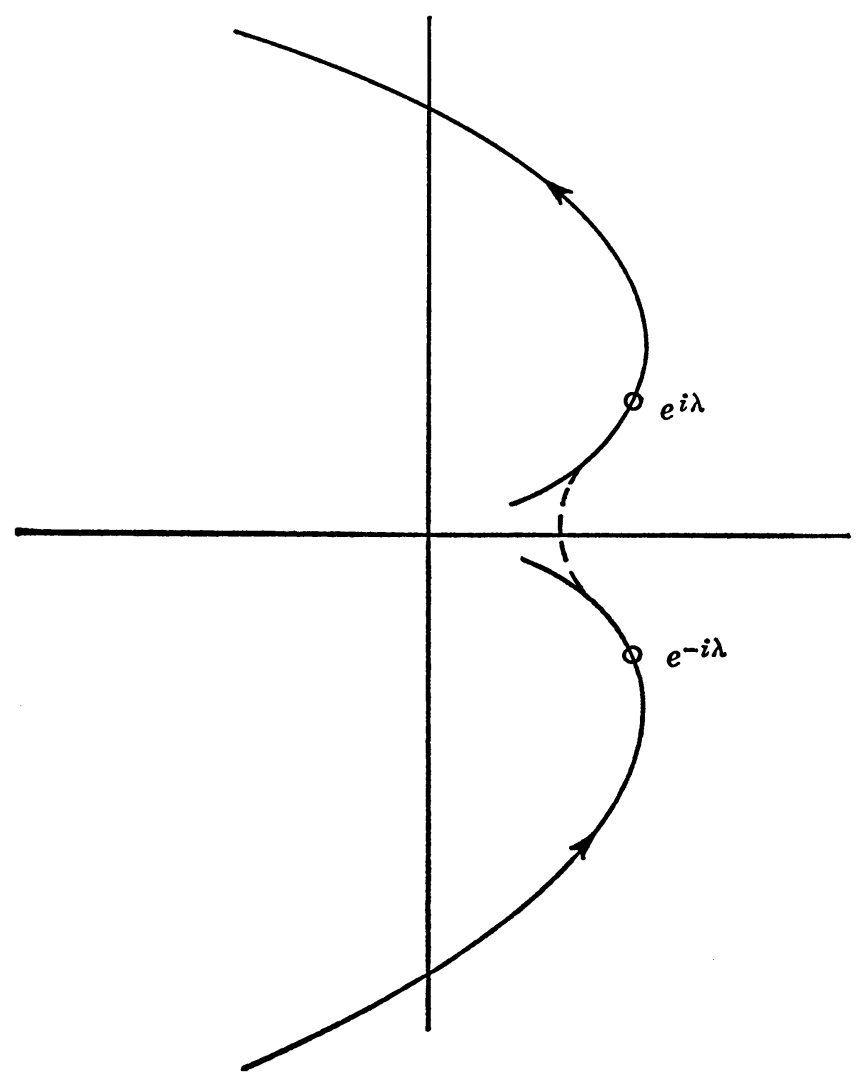

Figure 1

Steepest Descent Path 
Appendix I. The asymptotic expressions for

$$
I(\xi \nu)=\int_{0}^{\xi \nu} J_{\nu}(x) d x
$$

for $\xi$ fixed, $\nu \rightarrow \infty$, do not seem to be readily available in the literature, and so we give them here. They are easily obtained by the saddle point method [3].

For $\xi<1$, we write

$$
J_{\nu}(x)=\frac{1}{2 \pi i} \int_{C} \frac{d t}{t^{\nu+1}} e^{x / 2(t-1 / t)}
$$

where $C$ is a contour starting at $t=-\infty$, circling the origin once counterclockwise, and returning to $-\infty$. Integration yields

$$
I(\xi \nu)=\frac{1}{2 \pi i} \int_{C} \frac{d t}{\frac{1}{2}\left(t^{2}-1\right)}\left\{e^{\nu[\xi / 2(t-1 / t)-\ln t]}-1\right\} .
$$

Take $C$ to lie outside the points $t= \pm 1$; the second term then vanishes. To evaluate the first term asymptotically, for large $\nu$, choose the saddle point at $t_{0}=e^{\lambda}$ (where sech $\lambda=\xi$ ), and deform $C$ to pass through this point. The result is found to be

$$
I(\xi \nu) \sim \frac{1}{(2 \pi \nu \tanh \lambda)^{1 / 2}} \cdot \frac{\exp \{-\nu(\lambda-\tanh \lambda)\}}{\sinh \lambda} .
$$

Secondly, let $\xi>1$, and set $\cos \lambda=1 / \xi$. Again we use the contour $C$ of Eq. (I-3), but now $C$ must pass through the two saddle points at $t_{0}=\exp ( \pm i \lambda)$. The steepest descent paths are as shown in Fig. (I); $C$ coincides with them, except for the dotted line which transfers from one to the other. Because of the residue at $t=1$, the second term no longer vanishes; evaluating the first asymptotically, and putting the two terms together, we obtain

$$
I(\xi \nu) \sim 1-\left(\frac{2}{\pi \nu \tan \lambda}\right)^{1 / 2} \cdot \frac{\cos \{\nu(\tan \lambda-\lambda)+\pi / 4\}}{\sin \lambda} .
$$

University of Washington

Department of Aeronautics and Astronautics

Seattle, Washington 98105

1. R. Courant, K. Friedrichs \& H. Lewy, "Über die Partiellen Differenzengleichungen der Mathematischen Physik," Math. Ann., v. 100, 1928, pp. 32-74; English transl., IBM J. Res. Develop., v. 11, 1967, pp. 215-234. MR 35 \#4621.

2. Milton Abramowitz \& I. A. STEGUN (Editors), Handbook of Mathematical Functions with Formulas, Graphs, and Mathematical Tables, Nat. Bur. Standards Appl. Math. Series, no. 55, Superintendent of Documents, U. S. Government Printing Office, Washington, D. C., 1964, pp. 366-367; 3rd printing, with corrections, 1965. MR 29 \#4914; MR 31 \#1400.

3. G. Carrier, M. Krook \& C. Pearson, Functions of a Complex Variable: Theory and Technique, McGraw-Hill, New York, 1966, p. 257 ff. MR 36 \#5308.

4. H. O. KREISS \& E. LUNDQUIST, "On difference approximations with wrong boundary values," Math. Comp., v. 22, 1968, pp. 1-12. MR 37 \#3777.

5. M. Y. T. APELKRANS, "On difference schemes for hyperbolic equations with discontinuous initial values," Math. Comp., v. 22, 1968, pp. 525-539. 